\title{
Mobile health screening initiatives: a narrative of three unique programs in underserved populations
}

This article was published in the following Dove Press journal:

Innovation and Entrepreneurship in Health

17 July 2015

Number of times this article has been viewed

\author{
Pedram Daraei' \\ Charles E Moore ${ }^{1-3}$ \\ 'Department of Otolaryngology, Head \\ and Neck Surgery, Emory University, \\ ${ }^{2}$ Grady Health System, ${ }^{3} \mathrm{HEALing}$ \\ Community Center, Atlanta, GA, USA
}

Correspondence: Pedram Daraei;

Charles E Moore

Department of Otolaryngology,

Head and Neck Surgery, Emory

University Hospital, 550 Peachtree

Street NE, Medical Office Tower,

I I th Floor, Atlanta, GA 30308, USA

Tel +l 404778338 I

Fax + I 4047784295

Email cemoore@emory.edu
Background: Comprehensive screening mechanisms are critical and must be utilized in an appropriate fashion in populations with a presumed high prevalence of disease, requires low cost of screening, and available and effective treatment modalities. Alarmingly, racial and socioeconomic disparities in medical screening programs remain vast and ultimately contribute to poorer outcomes. Improving screening in areas of lower socioeconomic status extends a service to individuals who may have otherwise gone undiagnosed, in areas where disease is often diagnosed as late-stage disease, accompanied by comorbid conditions.

Methods: The authors coordinated and implemented three unique mobile health initiatives throughout underserved populations in metropolitan Atlanta. For each health initiative, a corresponding review of the English literature was performed using PubMed/MEDLINE. Special attention was paid to minority populations. Reference searches of the retrieved articles were performed manually to ensure that all available studies and data were reviewed.

Results: Mobile health screening was performed in three ways. The first focused on hypertension and asthma by screening individuals at a location commonly visited, ie, the grocery store. The second targeted obesity in underserved populations through a simple identification program that educated individuals on foods that are healthy and those that are not. This was performed in grocery stores, which we consider the "frontline" of nutrition-based decisions. Lastly, we developed an educational program targeting tobacco products, particularly e-cigarettes, which we implemented for adolescent populations through our metropolitan area.

Conclusion: Mobile screening is the first step in a facet of prevention that goes beyond traditional "in-office" screening. Targeting specific populations is of utmost importance, and engaging individuals at the community level can greatly improve the likelihood of success, particularly if the health care practitioners involved understand the cultural characteristics and customs of that population. Engaging health care practitioners in mobile screening represents a significant previously untapped resource that can increase screening throughput and greatly improve outcomes for patients who would otherwise go with an undiagnosed disease process.

Keywords: mobile, health screenings, underserved, obesity, tobacco, food insecurity, disparity, e-cigarettes

\section{Introduction}

Modern advances in the medical field have exponentially increased the diagnostic abilities of medical practitioners over the past half century, while simultaneously decreasing the morbidity of what were once considered fatal diseases. Advances in medicine are also changing the way in which medicine is practiced, moving from a reactionary method of patient care toward a more preventive approach. Preserving 
health prior to the onset of potentially avoidable medical illness is essential and critical in an effort to reduce morbidity and increase quality of life for patients.

In order to prevent disease, a comprehensive screening tool must be utilized. A tenet of effective screening is to employ a screening tool for detection of disease that has high prevalence. For this reason, we targeted three specific disease processes for which we could develop mobile screening programs, ie, hypertension, obesity, and tobacco use. Furthermore, a high racial disparity exists between African American and white populations, with respect to the aforementioned disease processes. ${ }^{1-5}$

Our goals included the following: to extend the mobile service to individuals who would otherwise not receive general health screening, to educate individuals in the underserved community, and take steps to eliminate the racial disparity that exists in underserved communities. We accomplished these initiatives through three activities, each with a unique goal, but with the underlying themes of education and prevention.

\section{Materials and methods}

The authors coordinated and implemented three unique mobile health initiatives in underserved populations in metropolitan Atlanta. All of these screening events, although they may have entailed different disease processes, required similar steps to be successful. A general summary is included in Figure 1, which presents the steps needed to perform successful health screening.

For each of the three health initiatives discussed in this manuscript, a corresponding review of the English literature was performed using PubMed/MEDLINE. Special attention was paid to minority populations. Keywords used included, but were not limited to, the following: "hypertension", "obesity", "screening", "tobacco", "adolescent", "e-cigarettes", and "mobile". Reference searches were performed manually for all retrieved articles to ensure that all available studies and data were reviewed. Each health screening initiative was first reviewed and approved by the Emory University Institutional Review Board.

\section{Results}

\section{Mobile health screening}

Our first mobile health screening initiative targeted hypertension and asthma. With the help of a grocery store located in a high-density, underserved community of metropolitan Atlanta, we were able to organize a 2-hour event offering screening to customers in the store.
Individual stations were created, one with a sphygmomanometer, one with an asthma questionnaire, and one with a binocular eye examination machine. As individuals came into the store, they were asked if they would like to participate in a free health screening check. Family and friends were often present, allowing simultaneous shopping and screening. A physician was present at all times to supervise the screening process and to verify that the volunteers performed proper medical technique for each medical and health screening.

A healthy eating demonstration also attracted individuals to the stations. In this setting, the use of visual and olfactory stimulation was utilized as a tool to create interest on the part of the potential screening participants. A professional chef volunteered her time to demonstrate healthy cooking alternatives using fruit, vegetables, and other non-processed ingredients. The recipe was handed to the participants, giving them a quick, easy, and delicious meal that they could replicate at home.

As participants were screened, abnormal values were noted and the participant was told of the abnormal value. They were then referred to an income-based clinic, which provides medical services based on a sliding fee cost schedule based on the individual's income, for a comprehensive workup with medical management and long-term follow-up. Lastly, the participant was encouraged to see their primary care physician regularly for routine screening. If the participant did not have a primary care physician, they were again referred to a nearby income-based clinic. Throughout the process, the participant was able to obtain basic information on their health status, learn fundamental information about their disease process, and, if applicable, be supplied with information on resources for care in their community, and obtain a healthy snack in the process.

\section{The Nutrition Initiative}

The Nutrition Initiative is a program implemented by the senior author, an otolaryngologist at our public metropolitan hospital system and founder of the HEALing Community Center. Our design was simple, ie, a sticker system indicating preferred healthier food choices that an individual might purchase. The initial design was created employing three colors, similar to those at traffic lights. A green star represents food of the "best" nutritional quality, such as fresh fruit and vegetables. A yellow star represents food of "good" nutritional quality. A red star indicates products of "bad" nutritional quality that are harmful to one's health and should be avoided, such as tobacco products. A sticker 
Preliminary planning (3-6 months in advance depending on size of event)

1. Discuss and obtain approval from location to hold health screening

2. Select point person/chairman for health event

3. Select additional members to assist with planning and logistic execution of health event

4. Decide on a health area of focus (if applicable)

5. Discuss rules, requirements, and expectations of location, clients, and staff

6. Confirm date and potential "rain date" (ie, alternative date)

7. Designate a contact person

Health event development (2-3 months in advance depending on size of event)

1. Create publicity (if appropriate)

a. News releases, posters, flyers, radio public service announcement spots

2. Create flyers/handouts (if appropriate)

a. Attendee record forms

b. Permission slips (if appropriate)

c. Signs for health stations

d. Health referral information and forms

e. Transportation options for referrals

3. Oversee equipment, location, operation

a. Check on participating service provider requirements

b. Ensure all equipment is in working order

c. Review logistical issues

4. Organize volunteers/staff

5. Prepare for set up and clean up

6. Develop a timeline to check status of health event

Pre-event (1-2 months in advance depending on size of event)

1. Distribute flyers/handouts

2. Ensure adequate equipment is in place and functional

3. Recruit additional volunteers/staff if needed

4. Plan refreshments for volunteers/staff

Day of event

1. Set up health stations

2. Have name tags for volunteers/staff

3. Sign in volunteers (name, email, mailing address, phone number)

4. Assign 1-2 people as "go to" people to troubleshoot during the event

5. Address issues that may arise during the event and document in order to make adjustments for future events

6. Clean up location

After the event

Figure I Steps for a successful health screening event.

was placed next to the price label for each item so that it could be noticed easily. A large poster was then created that could quickly explain the idea of the sticker system at a glance, and was displayed near the entrance to the food section of the store. It is important to note that this system was designed to primarily focus on the positive food choices available for purchase.

Our first event was launched at a Walmart store (Bentonville, AR) in an underserved, high-density area near downtown Atlanta. Nearly 50 volunteers, including 
community members, physicians, and medical, dietetic, and undergraduate students, participated and labeled each foodrelated aisle in the store in less than 3 hours. Tobacco products scattered throughout the store were also found and labeled red to indicate that they should be avoided. Data were collected to allow for evaluation and dissemination of our initiative and to monitor the effectiveness of the program.

The next goal was to increase awareness of the Nutrition Initiative. To combat poor health in the community, we decided to target the parent-child unit. This endeavor would increase the pervasiveness of our message in the community and improve the likelihood that could survive and this knowledge persist through a generation of individuals in a family. The most effective approach to accomplish this was in the school setting, specifically at meetings of the ParentTeacher Association. Our aim here was to notify parents, teachers, and students of the sticker system, and to convey our ideas to children at a young age. At these events, health screenings for diabetes, hypertension, and other conditions were performed in conjunction with healthy cooking demonstrations to illustrate that making small changes over time can improve one's overall health.

As a second stage in the shelf-labeling program, we have developed an alternative system in conjunction with the Emory Urban Health Initiative community, and philanthropic organizations. A modification of this labeling system includes the development of a Food Oasis Program label. This current labeling system focuses only on positive food choices. A Food Oasis Program label is placed on the better and best food item choices, thereby drawing the attention of the shopper to those items as opposed to providing a showcase for poor choices.

Analyses of the effectiveness of these two versions of the labeling systems are currently in process, but the Nutrition Initiative pilot program has been successful, and future additional labeling efforts will take place at supermarkets in high-density underserved areas throughout metropolitan Atlanta. As the Nutrition Initiative expands, and we are able to compile data, we anticipate that the sticker system will be further modified to continue improved identification of healthier food options and also to ensure lasting effects.

\section{E-cigarettes and the ASHES curriculum}

In an effort to reduce tobacco use in the adolescent population, including smokeless tobacco in the form of electronic cigarettes, we created a curriculum to provide information to students and families in underserved areas of our community.
In conjunction with the Rollins School of Public Health at Emory University, we created a pilot program called the Anti-Smoking Health Education Series (ASHES) curriculum, consisting of six lessons, lasting 20-30 minutes each, intended for elementary school students aged 6-11 years. The goal was to institute a simple, reproducible, and sustainable learning program that would decrease tobacco use in the adolescent population.

The curriculum was worksheet-based and required only paper and pencil. In addition, the worksheets were easily transferable - in other words, no special training was necessary to teach the curriculum. The six lessons progressed through identification of tobacco products, the effects of tobacco, peer pressure, smoking and the environment, tobacco in the media, and ended with a smoking-free pledge. At the end of each lesson, a review activity re-engaged the participants and also assessed their knowledge of the lesson that had just been imparted to them. The assessments were twofold, ie, to recapitulate important terms and ideas and to create an objective data set so that the effectiveness of the curriculum could be measured.

After the session, the data from the activities was reviewed and demonstrated an overwhelmingly positive gain in the fund of knowledge by the participants. One hundred percent of the adolescent participants knew that cigarettes were addictive, $80 \%$ knew that nicotine was the addictive compound, $90 \%$ knew that cigarettes contained contaminants such as tar and ammonia, and $90 \%$ associated peer pressure with tobacco use. Reassuringly, $100 \%$ of the participants were confident that they knew three or more facts that they could discuss with family to help family members stop smoking.

During the presentations and discussions arising from these sessions, it was determined that there was a need to incorporate a discussion of the use of marijuana in the curriculum. These sessions became a safe place for elementary students to not only be educated on tobacco products but also share their personal experiences. Consequently, an additional component of the curriculum following the same format is being created by the Morehouse Public Health Program focused on marijuana use and its effects. This collaborative effort between academic institutions demonstrates how health care practitioners can be effectively engaged in activities to greatly improve outcomes by working together in a collective fashion. 


\section{Discussion}

In order to be as effective as possible in our mobile health screening initiative, we targeted the most common disease processes in the general population and focused on conditions that could be easily screened for in the community setting, such as hypertension, asthma, and obesity. We also targeted one of the most highly impactful determinants of health, ie, tobacco.

\section{Mobile health screening}

Key to performing community-based interventions such as mobile screenings is providing an environment where individuals are capable of having the screening performed in an easy and proficient fashion. It is critical to engage people in a physical area and in a manner where they can feel safe, their privacy will not be compromised, and in an environment where the screening can be repeated at any time. On occasion this requires modification of the location to provide the security required. One example of a location utilized by the authors of this paper that was modified to create the appropriate environment was a supermarket in an underserved region of our metropolitan area. This site is commonly used by the community and draws a high number of people.

Costs were kept to a minimum, with the goal of reproducibility and transferability without the need for great expense. The supermarket provided basic materials including chairs and tables. The volunteers, who consisted of medical students and physicians, provided the necessary medical supplies. The overall cost to the team for this exercise was approximately $\$ 5$, due primarily to the cost of developing flyers to advertise the event.

\section{Nutrition Initiative}

The Nutrition Initiative was developed in an effort to encourage people to think about health in the broader sense. Health is intimately connected to social determinants. Social determinants of health are the precursor social and economic factors that influence health and disease. This includes

1). "Within the past 12 months we worried whether our food would run out before we got money to buy more" and

2). "Within the past 12 months the food we bought just didn't last and we didn't have money to get more".

Figure 2 Two-item food insecurity screen. the conditions in which we live, work, and learn, ${ }^{6}$ and are effectively the root determinants of health and disease. The majority of chronic disease conditions are influenced by poor nutrition and a sedentary lifestyle..$^{7-11}$ Therefore, use of a validated nutrition screening mechanism, such as a brief questionnaire (Figure 2) ${ }^{12}$ in a mobile setting is valuable in the prevention of disease. The Nutrition Initiative screens for signs of food insecurity in a mobile fashion in an effort to eliminate poor eating habits and promote healthy preventative measures by making small and stepwise changes in the community. Our goal is to create a simple but effective way to identify healthy foods at supermarkets and grocery stores in underserved areas. Ideally, anyone who walks into a grocery store would be able to select a healthy item easily by looking at the labeling.

Screening initiatives can be implemented in several forms. Creativity is paramount in constructing situations that continually pique interest and engage individuals in topics that are important to their health. Nutrition is integral to good health, and a healthy diet portends less morbidity and mortality. Fortunately, American culture is becoming more and more health-conscious and, as a result, "fast food" corporations are moving toward healthier menu options. These corporations have drastically altered their marketing campaigns, and their message is slowly being disseminated throughout the community. Individual states are following suit, with 867 nutrition-based legislative bills introduced or enacted since $2011 .{ }^{13}$ Georgia ranks 14th among the states, with 17 acts of legislation to further cultivate constructive and progressive health and nutrition enterprises. ${ }^{13}$ Although government agencies are making strides toward healthy eating habits, a significant education gap remains in the general population that must be overcome with health initiatives such as the Nutrition Initiative.

\section{E-cigarettes and the ASHES curriculum}

The lack of information on the use of tobacco products led to the development of youth surveys to assess what future generations understood about the effects of use of tobacco products. As these screening surveys were being given to youth in community settings during screening events, it was identified that e-cigarette use was also becoming popular. E-cigarette use in the US adolescent population has increased nearly twofold over the past several years. ${ }^{14,15}$

Many trends and associations have been found after analyzing demographic data of adolescent e-cigarette users. Of current e-cigarette users, $75.0 \%$ were traditional cigarette smokers. ${ }^{16}$ 
However, 9.2\% were never traditional cigarette smokers, demonstrating that one in 10 e-cigarette smokers are not using e-cigarettes as an alternative or replacement for cigarettes; instead they are likely naïve to traditional cigarettes but are now addicted to their smokeless counterparts.

One of the more concerning aspects of e-cigarettes is the increasingly widespread use of advertisements. Historically, the paradigm of traditional cigarette advertisements has changed immensely. Advertisements were predominantly found in magazines and newspapers, transitioned to television, and were ultimately banned by the Federal Communications Commission. Unfortunately, advertisements for e-cigarettes remain unregulated. Companies such as The Neilsen Company (New York, NY), which tracks ratings for television programs and networks, estimates that youth exposure to television e-cigarette advertisements has increased $256.0 \%$ from 2011 to $2013 .{ }^{17}$ The four networks that advertise e-cigarettes most frequently, in descending order, are AMC (New York, NY), Country Music Television (Nashville, TN), Comedy Central (New York, NY), and WGN (Chicago, IL). Unfortunately, there appears to be no structured opposition to e-cigarette advertisements, as there is with advertisements for traditional tobacco products.

Ultimately, the increasing prevalence of e-cigarette use is concerning due to the possibility of harm caused by contaminants to users. There are multiple contaminants that are of concern, including polycyclic aromatic hydrocarbons, tobacco-specific nitrosamines, and volatile organic compounds, all of which are found in traditional cigarettes, although they are found in much lower concentrations in e-cigarettes when compared with traditional cigarettes. ${ }^{18}$ However, current comprehensive data on this subject remain controversial and minimal at best. After implementation of the ASHES curriculum, it is clear that adolescents respond to our educational intervention in the short term. However, a long-term decrease in the prevalence of use of tobacco, marijuana, and e-cigarette would be the true measure of effectiveness and will be measured in the future. Due to its reproducibility and low-cost, the ASHES curriculum can also be implemented easily in other underserved communities. In doing so, we can make efforts to prevent adolescent tobacco, marijuana, and e-cigarette use, and avoid the long-term morbid complications.

\section{Conclusion}

By extending mobile health screening programs into underserved areas, we have provided a much needed service to populations that often harbor significant undiagnosed or under diagnosed disease burden. Many chronic disease processes can be appropriately identified, and individuals can then be directed to treatment facilities that can manage these conditions in the long-term setting. In doing so, we avoid delayed diagnoses, and may then decrease costs to both individual patients and society.

Mobile screening can be the first preventative step toward avoiding complicated sequelae or morbidities associated with late-stage disease. To increase effectiveness, it is important to ensure a close association with the target population by understanding tendencies, cultural characteristics, and the likelihood of a positive response to screening initiatives. Medical practitioners who work in areas that serve the target population can achieve this best. Practitioners who are established in the community in which they are screening can reach patients in a setting that is comfortable for both the practitioner and patient, which will facilitate effective screening and engage the patient in unique methods and opportunities to manage important aspects of their lives.

Increasing involvement on the part of practitioners, including primary care physicians, subspecialty physicians, dentists, and advanced practice providers, can increase the volume of patients screened, which will ultimately improve the ability of such a mobile screening program to identify patients harboring the disease being screened for. Engaging medical practitioners in mobile screening programs is critical, as they represent a previously untapped resource of power that can greatly improve prevention, particularly in underserved populations. In the USA, we spend nearly $\$ 3$ trillion on health care expenses, but our health care outcomes are not equivalent to our exorbitant expenses. ${ }^{19}$ An increased focus on prevention and increasing involvement of medical care practitioners in mobile screening programs may improve this worrisome trend.

\section{Disclosure}

The authors report no conflicts of interest in this work.

\section{References}

1. Moore CE, Warren R, Maclin SD Jr. Head and neck cancer disparity in underserved communities: probable causes and the ethics involved. $J$ Health Care Poor Underserved. 2012;23(4 Suppl):88-103.

2. Rereddy SK, Jordan DR, Moore CE. Dying to be screened: exploring the unequal burden of head and neck cancer in health provider shortage areas. J Cancer Educ. November 26, 2014. [Epub ahead of print.]

3. Flegal KM, Carroll MD, Kit BK, Ogden CL. Prevalence of obesity and trends in the distribution of body mass index among US adults, 1999-2010. JAMA. 2012;307(5):491-497.

4. Ogden CL, Carroll MD, Curtin LR, Lamb MM, Flegal KM. Prevalence of high body mass index in US children and adolescents, 2007-2008. JAMA. 2010;303(3):242-249. 
5. Rossen LM, Schoendorf KC. Measuring health disparities: trends in racial-ethnic and socioeconomic disparities in obesity among 2- to 18-year old youth in the United States, 2001-2010. Ann Epidemiol. 2012;22(10):698-704.

6. The Conversation. What are "social determinants of health?" November, 2012. Available from: http://theconversation.com/what-are-socialdeterminants-of-health-10864. Accessed November 20, 2012.

7. Cupisti A, D'Alessandro C, Morelli E, et al. Nutritional status and dietary manipulation in predialysis chronic renal failure patients. $J$ Nutr. 2004;14(3):127-133.

8. Schulze MB, Hoffmann K, Kroke A, Boeing H. Risk of hypertension among women in the EPIC- Potsdam Study: comparison of relative risk estimates for exploratory and hypothesis-oriented dietary patterns. Am J Epidemiol. 2003;158(4):365-373.

9. Sesso HD, Cook NR, Buring JE, Manson JE, Gaziano JM. Alcohol consumption and the risk of hypertension in women and men. Hypertension. 2008;51(4):1080-1087.

10. Al-Sinani M, Min Y, Ghebremeskel K, Qazaq HS. Effectiveness of and adherence to dietary and lifestyle counseling: effect on metabolic control in type 2 diabetic Omani patients. Sultan Qaboos Univ Med J. 2010;10:341-349.

11. DAFNE Study Group. Training in flexible, intensive insulin management to enable dietary freedom in people with type 1 diabetes: Dose Adjustment For Normal Eating (DAFNE) randomised controlled trial. BMJ. 2002;325:746.

12. Hager ER, Quigg AM, Black MM, et al. Development and validity of a 2-item screen to identify families at risk for food insecurity. Pediatrics. 2010;126(1):e26-e32.
13. Centers for Disease Control and Prevention. Chronic Disease State Policy Tracking System. March, 2013. Available from: http://apps. nccd.cdc.gov/CDPHPPolicySearch/Default.aspx. Accessed March 15, 2013.

14. Dutra LM, Glantz SA. Electronic cigarettes and conventional cigarette use among US adolescents: a cross-sectional study. JAMA Pediatr. 2014;168(7):610-617.

15. Centers for Disease Control and Prevention. Notes from the field: electronic cigarette use among middle and high school students United States, 2011-2012. MMWR Morb Mortal Wkly Rep. 2013;62(35):729-730

16. Camenga DR, Kong G, Cavallo DA, et al. Alternate tobacco product and drug use among adolescents who use electronic cigarettes, cigarettes only, and never smokers. J Adolesc Health. 2014;55(4):588-591.

17. Duke JC, Lee YO, Kim AE, et al. Exposure to electronic cigarette television advertisements among youth and young adults. Pediatrics. 2014;134(1):e29-e36.

18. Burstyn I. Peering through the mist: systematic review of what the chemistry of contaminants in electronic cigarettes tells us about health risks. BMC Public Health. 2014;14:18.

19. National Health Expenditures. 2013 Highlights. Available from: http://www.cms.gov/Research-Statistics-Data-and-Systems/StatisticsTrends-and-Reports/NationalHealthExpendData/downloads/highlights pdf. Accessed May 5, 2014
Innovation and Entrepreneurship in Health

\section{Publish your work in this journal}

Innovation and Entrepreneurship in Health is an international, peer reviewed, open access journal publishing original research, reports, reviews and commentaries on innovation and entrepreneurship in health. Special focus will be given to the theory, process, and practice of innovation and entrepreneurship by individuals and organizations

\section{Dovepress}

within the health care context globally. The manuscript management system is completely online and includes a very quick and fair peer review system, which is all easy to use. Visit http://www.dovepress.com/ testimonials.php to read real quotes from published authors.

Submit your manuscript here: http://www.dovepress.com/innovation-and-entrepreneurship-in-health-journal 